Itinéraires Itinéraires

Littérature, textes, cultures

Numéro inaugural | 2008

L'homme en tous genres

\title{
L'écriture polymorphe de la masculinité chez Rabelais
}

David LaGuardia

\section{OpenEdition}

1 Journals

Édition électronique

URL : http://journals.openedition.org/itineraires/2205

DOI : 10.4000/itineraires.2205

ISSN : 2427-920X

Éditeur

Pléiade

Édition imprimée

Date de publication : 1 décembre 2008

Pagination : 49-61

ISBN : 978-2-296-07519-1

ISSN : 2100-1340

Référence électronique

David LaGuardia, "L'écriture polymorphe de la masculinité chez Rabelais », Itinéraires [En ligne], Numéro inaugural | 2008, mis en ligne le 01 décembre 2008, consulté le 10 décembre 2020. URL http://journals.openedition.org/itineraires/2205; DOI : https://doi.org/10.4000/itineraires.2205

\section{(c) $(7)$}

Itinéraires est mis à disposition selon les termes de la licence Creative Commons Attribution - Pas d'Utilisation Commerciale - Pas de Modification 4.0 International. 


\title{
L'écriture polymorphe de la masculinité chez Rabelais
}

\begin{abstract}
The polymorphous writing of masculinity in Rabelais's works assumes at least two forms : first, apostrophes enunciated by a masculine subject who calls out to other men through affectionate insults written in a comic register, in order to allay the anxieties that the speaking subject has concerning his gender; and secondly, verbal improvisations based on the idea or image of the male body's private parts as they are made manifest in external signs, such as the beard and the codpiece, meant to be read and recognized by others of both sexes. Rabelais's writing of masculinity is hence discursive and intersubjective, and situates the "being" of masculine subjects within the incessant mode of semantic displacement that is characteristic of his works.
\end{abstract}

Le désir d'analyser la représentation de la masculinité chez Rabelais, et ses conséquences formelles et linguistiques, vient d'une perception fondamentale que l'on a trop souvent ignorée: ce n'est pas simplement comme médecin, moine, érudit, humaniste, évangélique, farceur, etc., que Rabelais écrit, mais aussi et peut-être surtout en tant qu'homme'. À chaque page de ses œuvres, le lecteur influencé par la pensée féministe est frappé par une voix narrative qui parle souvent, et parfois de manière obsessive, de sa masculinité. Mais qu'est-ce que cela signifie exactement? et comment prouver que le texte rabelaisien est en partie structuré par l'affirmation claire et explicite de l'appartenance de son auteur au sexe masculin?

La première étape de cette analyse de l'écriture masculine chez Rabelais consistera à définir ce que l'on entend par l'adjectif masculin dans ce contexte, et par le substantif développé plus tardivement dans l'histoire à partir de celui-ci. Même si à la Renaissance l'idée de la masculinité comme nous l'entendons aujourd'hui et le mot qui la désigne n'existaient pas,

1. Je remercie vivement Gary Ferguson et Mathilde Sitbon de leurs suggestions qui m'ont beaucoup aidé à formuler l'argument de cet article. 
une lecture attentive des œuvres de Rabelais révèle un écrivain préoccupé par la définition de ce qui constituait un homme et son rôle dans la société de l'époque. Comment décrire cette série de qualités, d'attitudes et de pratiques matérielles et discursives qui rendaient possible l'identification des hommes en tant qu'êtres sexués? Très brièvement, on pourrait définir la masculinité chez Rabelais comme une série d'expressions ou de figures textuelles divisées en plusieurs catégories. La masculinité dans l'œuvre de cet écrivain est un mode ou une manière de se référer à d'autres textes et à d'autres discours, une " posture » ou même une " pose » que le sujet masculin qui l'écrit et qui s'identifie comme tel adopte face aux demandes constantes des autres sujets masculins représentés dans le texte, parmi lesquels se situe, dans une position privilégiée, le lecteur, explicitement interpellé et « apostrophé » comme homme. Tous ces personnages masculins inscrits dans le texte «se regardent» constamment, afin de lire et de confirmer le sexe des autres et de s'assurer qu'ils appartiennent tous à la même catégorie d'êtres sexués.

Dès le début de l'œuvre rabelaisienne, le lecteur entend la voix d'un homme qui fait appel à d'autres hommes en affichant son être comme sujet masculin. Chez Rabelais il y a, par exemple, de nombreux appels et apostrophes à des lecteurs qui sont clairement caractérisés en fonction de leur appartenance au sexe masculin. Les célèbres « beuveurs tresillustres » du prologue du Gargantua pourraient servir d'exemple de ce type d'énoncé. Les expressions de cette première catégorie sont marquées par les particularités génériques de la grammaire française, ce qui pourrait peut-être soulever l'objection suivante, légèrement anachronique: le genre grammatical de la référence générique en français moderne est le masculin, de sorte que, du point de vue du lecteur d'aujourd'hui, cette apostrophe n'indique pas forcément le sexe des lecteurs présumés. Autrement dit, de ce point de vue, si le texte s'adresse aux «beuveurs tresillustres », et non aux «beuveuses tresillustres », cela ne veut pas dire que Rabelais, ou Alcofrybas son narrateur ${ }^{2}$, avait littéralement des hommes à l'esprit quand il écrivait et qu'il ne pensait pas aussi éventuellement à des lectrices ivres. Pourtant, on connaît la critique faite par la pensée féministe à ce genre d'argument: si la domination masculine a été tellement enchevêtrée dans tous les aspects de la culture, il n'est pas surprenant que l'usage du masculin pour signifier l'universel se soit imposé comme règle générale de la grammaire, avec la disparition du féminin que cet usage entraîne. Une lecture réfléchie de la littérature du passé doit tenir compte de cet effacement du féminin dans des procédés linguistiques et littéraires par lesquels les hommes parlent ou écrivent exclusivement à d'autres hommes, même si cette lecture risque d'être anachronique dans la mesure où l'usage du masculin pour désigner

2. Gérard Defaux nous rappelle les dangers de l'identification immédiate de Rabelais luimême avec la figure d'Alcofrybas. Voir Rabelais Agonistes : du rieur au prophète, Genève, Droz, 1997, p. 407-411. 
les deux sexes n'était pas aussi général à l'époque de Rabelais qu'il ne l'est aujourd'hui, la " féminisation » des noms de métiers (peintresses, physiciennes, médicineuses, etc.) ayant été courante. Ceci semble donc signifier que l'auteur s'adresse ici consciemment à des lecteurs qu'il conçoit comme des hommes. La suite de cette citation déterminante de Rabelais confirme cette première hypothèse: « Beuveurs tresillustres, et vous Verolez tresprecieux (car à vous non à aultres sont dediez mes escriptz) ${ }^{3}$. » On imagine mal qu'Alcofrybas s'adresse à des femmes dans cette dédicace.

La densité du discours rabelaisien se manifeste dans l'expression «Verolez tresprecieux », que l'on peut expliquer sur plusieurs niveaux à la fois. D'abord, il y a, bien sûr, le niveau parodique: un homme marqué par la vérole, nom populaire de la syphilis au $\mathrm{XVI}^{\mathrm{e}}$ siècle, ne peut être " précieux » que dans le contexte de la comédie où les excès de toutes sortes sont célébrés, surtout dans la littérature comique écrite par des clercs comme notre auteur. Dans cette littérature, il n'y a pas de fête sans boisson, fornication et adultère, justement les éléments évoqués dans le prologue du Gargantua. La sexualité débordante figurée par cette pratique comique, qui dépend évidemment de la participation des femmes, se communique par écrit dans les figures de l'apostrophe et de l'appel aux autres hommes ("à vous sont dédiés mes écrits»), mais elle implique en même temps l'effacement des femmes et du féminin. Un prolongement intéressant de ce registre comique est la transformation d'une marque négative - la marque de la syphilis, de la sexualité excessive - en marque positive qui établit une complicité entre la voix qui écrit le texte et ceux qui sont censés le lire, complicité qui prend le détour du sexe conçu comme maladie: vous et moi, lecteurs, sommes frappés par la même peste, ce désir débordant qui dépend, malheureusement, des femmes ${ }^{4}$. Les « Verolez » sont donc appelés à lire comme des hommes qui transgressent l'ordre social de la sexualité, limité au mariage, mais cette transgression est elle-même inscrite au cœur d'une convention comique qui permet aux hommes d'établir avec d'autres hommes les liens narratifs qui soutiennent leur conception d'eux-mêmes comme sujets masculins.

En second lieu, il y a chez Rabelais des séries de références à l'anatomie masculine et à son importance comme idée ou image qui contribuent à la formation d'une subjectivité textuelle masculine. L'idée du corps masculin sert de prétexte chez Rabelais à des improvisations linguistiques d'une virtuosité éblouissante, ce qui soulève une question essentielle à propos du statut de l'objet et du réel dans son œuvre. Quand on parle d'improvisations discursives qui prennent comme point de départ l'idée d'une

3. F. Rabelais, Euvres complètes, éd. M. Huchon, Paris, Gallimard, 1994, p. 5. Toutes les références renvoient à cette édition.

4. Dans son édition des Euvres complètes, Paris, Le Seuil, 1973, Guy Demerson interprète cette apostrophe initiale de la manière suivante: «Maître Alcofribas, médecin, s'adresse à des malades pour les guérir par le rire. » (p. 38) 
partie du corps masculin, comme par exemple les célèbres descriptions des braguettes du Gargantua ou du Tiers Livre, est-on autorisé à associer ces phénomènes textuels à l'idée d'un corps masculin réel et à une vraie masculinité qui serait à la fois un effet et une qualité de ce corps? Comme plusieurs critiques l'ont montré, le rapport entre ces descriptions souvent burlesques et le réel est incertain et vague ${ }^{5}$, et la plupart du temps il semble que Rabelais s'inspire plus d'autres textes et d'autres pratiques littéraires que d'une conception de la réalité. Il serait donc très problématique d'affirmer que les descriptions du corps masculin chez lui sont des indices d'une masculinité historiquement vérifiable et réelle; au contraire, il faudrait les concevoir comme une partie essentielle d'une pratique littéraire caractérisée par un déplacement sémantique et linguistique incessant. Autrement dit, la masculinité rabelaisienne doit être recherchée dans un procédé référentiel polymorphe qui ne se stabilise jamais.

Pour revenir à l'exemple des braguettes, il y a de nombreux passages comme le suivant, qui combine une apostrophe insultante aux lecteurs et une improvisation verbale centrée sur une partie déterminante de l'anatomie et des vêtements du petit Gargantua habillé par ses gouvernantes:

\footnotetext{
Et sabez quey hillotz, que mau de pipe vous byre, ce petit paillard tousjours tastonoit ses gouvernantes cen dessus dessoubz, cen devant derriere, harry bourriquet: et desjà commençoyt exercer sa braguette. Laquelle un chascun jour ses gouvernantes ornoyent de beaulx boucquets, de beaulx rubans, de belles fleurs, de beaulx flocquars: et passoient leur temps à la faire revenir entre leurs mains, come un magdaleon d'entraict. Puis s'esclaffoient de rire quand elle levoit les aureilles, comme si le jeu leurs eust pleu. L'une la nommoit « ma petite dille », l'autre « ma pine », l'autre « ma branche de coural », l'aultre « mon bondon, mon bouchon, mon vibrequin, mon possouer, ma teriere, ma pendilloche, mon rude esbat roidde et bas, mon dressouoir, ma petite andoille vermeille, ma petite couille bredouille. » (p. 35)
}

Voici un texte extraordinaire qui provoque une sorte de panique chez le lecteur moderne qui doit chasser le sens de tous ces mots dans des dictionnaires ou dans l'appareil critique de son édition. Il y a d'abord l'apostrophe en gascon au lecteur, que Mireille Huchon traduit de la manière suivante: « Et savez-vous quoi, les gars? Que le mal de tonneau vous fasse tourner! » (p. 35) Voilà un très bel exemple de ce qu'on appelle en anglais «male bonding », et que l'on pourrait peut-être traduire en français par « compagnonnage masculin », en faisant écho à l'expression « gentil compagnon » utilisée par Pantagruel quand il veut exprimer son affection pour son ami Panurge au chapitre Xv du Pantagruel. Chez Rabelais, ce genre d'appel

5. Cette idée a été réitérée dans le débat extensif autour du prologue du Gargantua, résumé dans mon article «French Renaissance Literature and the Problem of Theory: Alcofribas's Performance in the Prologue to Gargantua », EMF, n ${ }^{\circ} 10$, printemps 2005, p. 5-38. 
d'un sujet de l'écriture évidemment masculin à un sujet/lecteur aussi clairement masculin se fait souvent à travers une imprécation paradoxalement affectueuse. Ce même procédé textuel se trouve à la fin du prologue du Gargantua, dans une apostrophe insultante et extraordinairement vulgaire du point de vue du lecteur moderne: « Mais escoutez vietz d'azes, que le maulubec vous trousque! Mais vous soubvienne de boyre à my pour la pareille $^{6}[\ldots] . »($ p. 8) Il me semble clair que lorsque Alcofrybas s'adresse à ses lecteurs en les désignant comme des parties significatives de l'anatomie d'un animal mâle, il se présente comme un homme qui est en train de parler à d'autres hommes de l'identité sexuelle qu'ils partagent, se constituant ainsi comme un sujet qui s'identifie consciemment à son sexe. De la même manière, Rabelais active tout le vocabulaire festif conventionnel du vin et de la boisson quand il condamne les « gars » qui le lisent au « mal de tonneau ». Le vertige provoqué par le texte rabelaisien, comparé ici à l'idée de l'ivresse qui parcourt tout le passage de la braguette, peut se lire comme une sorte d'emblème des procédés textuels de cette œuvre étonnante. En fait, ce passage étourdit progressivement le lecteur par la série de références qui culmine dans la "(dé)nomination» du sexe du géant adolescent. En somme, l'écriture de la masculinité rabelaisienne est, entre autres choses, une manière de nommer le corps qui fait appel constamment aux autres hommes dans un registre comique afin de demander leur approbation pour un certain regard sur les relations entre les hommes, qui à son tour dépend d'une conception bien définie des femmes.

Il faut remarquer sans doute qu'il y a très peu de femmes dans le monde narratif de Rabelais et que les gouvernantes de ce passage ne servent qu'à motiver la description de la braguette de Gargantua. Les connotations des décorations apportées par les gouvernantes (bouquets, fleurs, rubans, guirlandes) sont assez claires : il faut que l'aspect extérieur de l'anatomie masculine représente son abondance reproductrice qui est comme «sollicitée » ici par les femmes. Cette notion de l'abondance obligatoire et nécessaire du sexe masculin était exprimée d'une manière encore plus exagérée dans la première description de la braguette au chapitre vIII du Gargantua: «Tousjours gualante, succulente, resudante, tousjours verdoyante, tousjours fleurissante, tousjours fructifiante, plene d'humeurs, plene de fleurs, plene de fruictz, plene de toutes delices ${ }^{7}$. ( (p. 25) Mais plus tard dans le passage du chapitre XI, le texte s'éloigne brusquement de ce registre « copieux » dans l'analogie du «magdaleon d'entraict» que les gouvernantes utilisent afin de « soigner» le petit géant. Selon Huchon,

6. Demerson explique cette expression de la manière suivante: « Vits d'ânes ; interpellation à la fois grossière et affectueuse ", édition citée en note 4, p. 41, n. 33 .

7. Dans sa lecture de ce passage, Terence Cave interprète les motifs floraux de la braguette comme des indices de sa fécondité qui la transforment en corne d'abondance, modèle de la copia rhétorique du texte rabelaisien. Voir The Cornucopian Text: Problems of Writing in the French Renaissance, Oxford, Oxford University Press, 1979, p. 183-187. 
cet objet est une « [p] etite masse de pâte, de forme cylindrique, utilisée pour faire des emplâtres » (p. 1096, n. 3). Ce mouvement métonymique à travers la décoration de la chose vers la chose elle-même la cache toujours sous cette comparaison médicale, qui transforme le membre masculin en « blessure » requérant les soins des gouvernantes, rappelant ainsi l'image du prologue de la masculinité comme maladie. Cependant, le procédé de déplacement sémantique ne s'arrête jamais dans cette œuvre: la braguette prend d'autres caractéristiques et « lève ses oreilles » entre les mains des femmes qui la décorent. Quelque part dans ce détournement constant on sait que Rabelais décrit un objet qui rend visible, en principe, le sexe du corps masculin, mais on aurait beaucoup de difficulté à dire exactement à quoi correspond cet obscur objet d'un « désir » qui est, au moins dans ce passage, féminin et riant («Elle est à moy, disoit l'une. - C'est la mienne, disoit l'aultre »), phénomène rarissime chez un écrivain qui en fait parle très peu de la « sexualité », surtout celle des femmes.

Rien que dans cette première partie du passage, l'objet privilégié que décrit Rabelais change de registre métaphorique quatre fois, se déplaçant du domaine sémantique des fleurs vers la référence médicale du magdaleon, ensuite vers le domaine des animaux, et enfin vers la série de mots qui désignent tous des objets phalliques utilisés pour pénétrer des choses diverses. Si le corps masculin est un objet « réel » représenté fréquemment chez Rabelais, et si l'on peut lire ce passage comme une description de l'excitation sexuelle du petit géant manipulé par ses gouvernantes, il est clair aussi que la représentation de ce corps comme objet est protéiforme et chimérique, puisqu'il ne prend jamais une forme stable. Le Gargantua est un livre particulièrement attentif à la formation, la discipline, l'éducation et l'habillement du corps du protagoniste, afin que celui-ci puisse se présenter proprement comme « prince humaniste », c'est-à-dire comme le modèle d'un homme superlatif de son époque ${ }^{8}$. Et à son tour, ce «dressage » du corps masculin nous invite à concevoir la masculinité du narrateur qui le décrit, au moins partiellement, comme un effet de cette série hallucinante de références à ce corps polymorphe. L'appartenance de Rabelais au sexe masculin réside justement dans cette façon problématique et instable de se référer aux corps des hommes, qui ne cesse jamais de se déplacer vers des registres sémantiques divers. Comme nous le verrons dans un instant au Tiers Livre, cette manière qu'ont les hommes de décrire leur propre corps a des conséquences importantes, non simplement pour l'élaboration de leur propre identité sexuelle ou genrée, mais aussi pour leur conception du monde social, spirituel et même « cosmique ».

8. La lecture d'Edwin Duval des livres de Rabelais se concentre largement sur leurs personnages fictifs, surtout Pantagruel, comme des incarnations du prince idéal et évangélique de la Renaissance. Voir, par exemple, The Design of Rabelais's «Pantagruel », New Haven, Yale University Press, 1991. 
Pour continuer l'analyse du même extrait, l'objet que la braguette est censée contenir semble disparaître dans le catalogue d'épithètes qui termine le passage. La description s'organise comme une sorte de réponse périphrastique à la question: « qu'est-ce que le membre viril, et comment peut-on le nommer dans un langage inadéquat à cette tâche? » Ici le discours rabelaisien atteint un de ces points culminants typiques de l'œuvre, et la lecture de l'énumération des noms prononcés provoque le vertige sémantique que j'ai déjà mentionné : « Ma petite dille, mon épingle, ma branche de corail, mon bondon, mon bouchon, mon vilebrequin, mon poussoir, ma tarière, ma pendeloque, mon rude ébat raide et bas, mon dressoir, ma petite andouille vermeille, ma petite couille bredouille. » Si l'on élucide ce catalogue à l'aide du Petit Robert et des notes de Demerson (p. 74) et d'Huchon (p. 35), la description du membre viril devient encore plus protéiforme : dille $=$ « fausset de tonneau »; bondon $=$ « bouchon de barrique »; vibrequin ou vilebrequin = « outil formé d'une mèche que l'on fait tourner à l'aide d'une manivelle coudée, et qui sert à forer, à percer des trous $» ;$ possouer $=$ " poussoir de sarbacane $»=\langle$ bouton sur lequel on appuie pour déclencher ou régler un mécanisme... tube creux servant à lancer de petits projectiles, par la force du souffle »; teriere ou tarière = " grande vrille pour percer des trous dans le bois » = « un outil formé d'une tige que termine une vis »; pendeloque $=$ « bijou suspendu à une boucle d'oreille; ornement suspendu à un lustre »; dressoir = « une étagère, buffet anciens où sont disposés des objets faisant partie du service de la table (vaisselle, récipients) ». Je cite toutes ces définitions afin de souligner la multiplicité changeante d'objets qui peuvent servir comme figures, substituts ou fétiches du membre viril dans l'imaginaire rabelaisien, hautement troublé par les manifestations oniriques d'un corps qui ne cesse jamais de s'enfuir dans ses déplacements linguistiques.

Ici on pourrait sans doute objecter que l'énumération de Rabelais est assez typique de la littérature comique de la fin du Moyen Âge et de la Renaissance, avec ses hyperboles et son goût pour les équivoques linguistiques. De ce point de vue, on pourrait dire que le comique du Gargantua n'est que le point culminant d'une littérature comique séculaire qui va des Facetiae et des fabliaux à travers les Cent nouvelles nouvelles et les nouvelles de Philippe de Vigneulles pour arriver aux œuvres de Rabelais. En ce sens, cet assemblage d'objets nommés sur la page n'est qu'une amplification hyperbolique des procédés utilisés pendant des siècles par les écrivains comiques de langue française. Tout en reconnaissant la vérité de cet argument, on peut aussi affirmer que Rabelais appartient à une longue tradition d'écriture masculine qui représente cette même idée du corps masculin dans des improvisations linguistiques à travers lesquelles les hommes entrent en contact avec d'autres hommes précisément en communiquant une version fantastique de l'anatomie masculine, toujours féconde et toujours désirée par des femmes complaisantes. Cette fantaisie, rédigée 
comme blague dans une apostrophe aux lecteurs masculins, est fortement marquée par le sexe de celui qui l'écrit. La fin du passage cité me semble aussi hautement significative de ce point de vue. Quand Rabelais nomme enfin les organes sexuels du géant, il le fait d'une manière intéressante: « ma petite andouille vermeille, ma petite couille bredouille ». Dans la virtuosité phonétique de ces mots, il y a peut-être l'idée que chez Rabelais on ne parle pas simplement du sexe masculin, mais c'est le sexe lui-même qui parle ou « bredouille », et à qui les gouvernantes parlent. Selon le Petit Robert, «bredouiller» signifie «parler d'une manière précipitée et peu distincte », tandis que Le Trésor de la langue française propose la définition « parler de façon précipitée et confuse, sans articuler ». Au début du $\mathrm{XVII}^{\mathrm{e}}$ siècle, « être en bredouille » signifie, pour Randle Cotgrave, « To be tipled, or tipsie ${ }^{9} »$ ( «être un peu éméché »). Dans un usage sans doute plus moderne, l'adjectif « bredouille » indique, encore selon le Robert, celui « qui n'a rien pris à la chasse ou à la pêche, ou qui n'a rien obtenu d'une entrevue, d'une démarche ». On a l'impression que chez cet auteur tellement troublant, le sexe masculin est toujours en train de bredouiller, mais comme c'est le cas du personnage qui incarne la masculinité rabelaisienne de la manière la plus frappante, c'est-à-dire le Panurge du Tiers Livre, le sujet masculin représenté dans ces pages revient toujours «bredouille» - les mains vides et souffrant du «mal de tonneau »-de ses expéditions en territoire féminin.

L'explication détaillée de cet extrait du Gargantua nous permet donc d'avancer les thèses suivantes: l'écriture de la masculinité chez Rabelais prend au moins deux formes, ou se présente sous deux modalités ou modes différents. D'abord, c'est l'apostrophe d'un sujet masculin qui fait appel à d'autres sujets masculins, souvent à travers des insultes affectueuses exprimées dans un registre comique. Deuxièmement, c'est une élaboration ou une improvisation verbale fondée sur l'idée du corps masculin et plus spécifiquement sur l'idée ou l'image du sexe masculin tel qu'il se manifeste visiblement dans des signes extérieurs censés être lus correctement par les hommes et les femmes, tout comme les gouvernantes « lisent » le corps de Gargantua dans le passage précédent. Dans une autre étude plus détaillée, j'ai montré que le Tiers Livre met en œuvre ce type d'écriture, ce qui ne veut évidemment pas dire que les éléments évangéliques et humanistes décrits par exemple par Edwin Duval dans son livre magistral sur ce texte ne sont pas présents ${ }^{10}$. Le discours rabelaisien est peut-être unique dans le

9. R. Cotgrave, A Dictionarie of the French and English Tongues (1611), Columbia, University of South Carolina Press, 1950. Ce sens rejoint évidemment le registre de la boisson et de l'ivresse qui parcourt toute l'œuvre de Rabelais.

10. D. LaGuardia, Intertextual Masculinity in French Renaissance Literature: Rabelais, Brantôme, and the "Cent nouvelles nouvelles", Aldershot et Burlington (Vermont), Ashgate, 2008; E. Duval, The Design of Rabelais's «Tiers Livre de Pantagruel », Genève, Droz, 1997. 
sens qu'il se développe simultanément sur plusieurs niveaux radicalement différents, étant à la fois, et dans les mêmes phrases, extrêmement vulgaire et extrêmement érudit. Un aspect de cette hybridité et de cette multiplicité qui a été moins étudié, à mon avis, est justement ce que je suis en train de signaler ici : l'écriture rabelaisienne comme exposition linguistique et polymorphe de la masculinité.

Le Tiers Livre représente l'exemple le plus extrême de l'écriture masculine chez Rabelais, qui atteint un point culminant d'obscurité aux chapitres XXVI-XXVIII, où Panurge et Frère Jean s'engagent dans un compagnonnage spectaculaire qui reproduit la structure discursive et « interpellatrice » ou " apostrophique » que j'ai déjà relevée entre le narrateur et ses lecteurs. Dans l'échange d'épithètes décrivant les organes sexuels du corps masculin qui constitue la plus grande partie de ces chapitres, cette manière d'exposer et de " dénommer » le corps des hommes implique les personnages mâles dans un rituel discursif de compagnonnage masculin qui a des caractéristiques bien connues: le récit d'un homme à un autre homme qui exagère au maximum sa propre puissance sexuelle, en même temps qu'il dénie cette même puissance aux autres hommes; l'exagération hyperbolique du désir des femmes pour les actes charnels; un appel aux autres hommes à partager la souffrance provoquée par la concupiscence démesurée des femmes, appel qui mène les hommes à établir un lien entre eux fondé sur ce qu'ils voient comme le partage inévitable des corps des femmes. Les longs catalogues de ces chapitres consacrés aux parties inférieures des hommes entraînent les personnages dans une exhibition de leur identité comme sujets masculins, ce qui signifie que les valeurs alternativement positives et négatives des deux catalogues, le blason et le contreblason ${ }^{11}$, dépendent de la manière dont Panurge comprend implicitement son propre être comme homme défini par rapport à sa conception fantastique des femmes.

Comme toutes les séquences du Tiers Livre, la description de cette camaraderie des deux personnages comme spectacle s'ouvre par la question fondamentale que Panurge pose à son ami Frère Jean: « dois-je me marier? » Cette demande d'un discours consolateur, adressée par le héros à son compagnon, commence d'une manière significative du point de vue de l'écriture de la masculinité: «Tien moy un peu joyeulx mon bedon. Je me sens tout matagrabolisé en mon esprit, des propous de ce fol endiablé [Her Trippa]. Escoute couillon mignon, couillon moignon [...]. » (p. 432) Ici l'amitié entre les hommes s'exprime à travers un discours qui «blasonne » le bas corporel des hommes ${ }^{12}$, y compris, bien sûr, le gros ventre (bedon).

11. Huchon remarque que ces chapitres s'inscrivent dans cette tradition particulière: «Rabelais fait ironiquement ici [ch. XXVI] un blason du corps masculin et en XXVIII compose un contreblason - tout comme Marot avait fait un contreblason du laid tetin », édition citée en note 3 , p. 1416, n. 1 .

12. Expression entendue au sens que lui donne M. Bakhtine, L'Euvre de François Rabelais 
Cette manière de blasonner les corps, à l'image des Blasons anatomiques du corps féminin, est en fait une des grandes marques d'un discours qui identifie celui qui le prononce comme sujet masculin. La réponse du moine à cette demande se situe au cœur d'une topographie cosmologique, scatologique et eschatologique de la masculinité, qui comporte presque l'univers entier:

Marie toy, de par le Diable, marie toy, et carrillone à doubles carrillons
de couillons. Je diz et entends le plus toust que faire pourras. Dés huy au soir
faiz en crier les bancs et le challit. Vertus Dieu à quand te veulx tu reserver?
Sçaiz tu pas bien, que la fin du monde approche? Nous en sommes huy plus
prés de deux trabutz et demie toise, que n'estions avant hier. L'Antichrist
est desjà né, ce m'a l'on dict. Vray est que il ne faict encores que esgratigner
sa nourrisse et ses gouvernantes: et ne monstre encores les thesaurs. Car il
est encores petit. Crescite. Nos qui uiuimus. Multiplicamini. Il est escript.
C'est matiere de breviaire. Tant que le sac de bled ne vaille trois patacz,
et le bussart de vin, que six blancs. Vouldrois tu bien qu'on te trouvast les
couilles pleines au jugement? dum uenerit judicare. (p. 434-435)

L'impératif fondamental de la masculinité, c'est-à-dire l'utilisation de l'anatomie masculine à des fins de procréation, s'inscrit entre les deux limites de l'univers chrétien, entre l'injonction de la Genèse qui commande aux hommes d'être « copieux » dans la reproduction et l'arrivée de l'Antéchrist avant le Jugement dernier. Dans un écho exquis à la scène du Gargantua que je viens d'examiner, l'avènement de cette figure diabolique est placé sous le signe d'une sorte de précocité sexuelle, quand il «égratigne » ses gouvernantes. Le bon fonctionnement de l'économie domestique, représentée ici par le blé et le vin, objets à la fois sacrés et festifs, est lié à l'activité génitale de l'homme. La copia obligatoire du corps masculin est exprimée par Frère Jean qui proclame que l'homme doit être infatigable dans son activité reproductrice afin d'assurer son propre salut. Ce passage évoque donc un schéma temporel, social et cosmologique centré sur le bas corporel masculin, dont la configuration discursive et intertextuelle sert de modèle aux autres parties de l'univers humain. Ainsi, quand un sujet masculin, vu comme le centre et le fondement du cosmos, souffre de doutes intellectuels, physiques et même métaphysiques concernant son rôle sexuel, comme c'est le cas pour Panurge tout au long du Tiers Livre, le monde qui l'entoure se transforme et se remplit de dilemmes qui ne trouvent pas de solution. Où qu'il cherche, Panurge n'entend que les deux possibilités irréconciliables : « marie toy, marie toy » (p. 434) ou « marie poinct, marie poinct» (p. 442). Dans une déformation sacrilège typique du comique de Rabelais, des cloches se balancent de droite à gauche, entre les impératifs positifs et négatifs, allégorie du balancement des testicules associé par Frère

et la culture populaire au Moyen Âge et sous la Renaissance, Paris, Gallimard, 1970, 1982 et 1990 . 
Jean à l'activité sexuelle obligatoire des hommes qui doivent « carrillone[r] à doubles carrillons de couillons ». Encore une fois, la virtuosité phonétique de Rabelais sert de prétexte au développement d'une version imaginaire du monde centrée sur le fonctionnement du sexe masculin.

Face à un doute et physique et métaphysique, Panurge demande conseil à son ami qui lui répond en faisant référence à un code de la masculinité à la fois sacré et scatologique. La deuxième partie de cette réponse concerne l'usage nécessaire de l'anatomie masculine, qui ne doit pas souffrir d'interruption: « Si continuellement ne exercez ta mentule, elle perdra son laict, et ne te seruira que de pissotiere: les couilles pareillement ne te serviront que de gibbessiere [...]. Pourtant fillol, maintien tout ce bas et menu populaire Troglodyte, en estat de labouraige sempiternel. » (p. 436) Comme je l'ai déjà signalé, une partie essentielle du compagnonnage masculin est le discours partagé entre deux hommes qui exagèrent leur virilité et soulignent la nécessité d'une activité sexuelle sans répit. Pour ces hommes qui regardent constamment les autres hommes afin de lire les signes de cette activité, les corps deviennent des surfaces topographiques qu'ils interprètent allégoriquement. Plus loin dans cette même scène, la barbe de Panurge, marque extérieure et visible de son sexe, est littéralement lue par Frère Jean comme une « mappemonde » :

Ta barbe par les distinctions du gris, du blanc, du tanné, et du noir, me semble une Mappemonde. Reguarde icy. Voy là Asie. Icy sont Tigris et Euphrates. Voy là Afrique. Icy est la montaigne de la Lune. Voydz tu les paluz du Nil? Deçà est Europe. Voydz tu Theleme? Ce touppet icy tout blanc, sont les mons Hyperborées. Par ma soif mon amy, quand les neiges sont es montaignes: je diz la teste et le menton, il n'y a pas grand chaleur par les valées de la braguette. (p. 438)

Dans cet exemple éblouissant d'écriture masculine, la tête de l'homme représente le monde entier, conçu du point de vue de l'humanisme érudit, dans un déploiement de connaissances qui relèvent à la fois de la « cosmographie » moderne et de la géographie de l'Antiquité. Dans ce procédé « masculiniste », un homme en regarde un autre qui l'a interpellé et lui répond avec l'affirmation discursive d'une vérité concernant le corps de son interlocuteur. La particularité et l'étrangeté de la masculinité rabelaisienne sont plus qu'évidentes dans ce passage et se manifestent en plusieurs étapes. D'abord, il y a le simple acte de la «lecture» d'un corps masculin par un homme qui le regarde. Deuxièmement, il y a le détour de cette lecture par l'érudition de l'époque, fortement influencée par la cartographie. Troisièmement, il y a le « rabaissement» du regard masculin qui interprète le corps de l'autre en lisant ses parties supérieures en termes du fonctionnement de ses parties inférieures. Quatrièmement, l'amitié masculine implique l'expression d'un doute moqueur sur la puissance sexuelle de l'autre, doute qui sera violemment rejeté par Panurge, comme nous le verrons par la suite. Enfin, ce discours masculin, qui comporte un savoir 
énorme, un mode de lecture et une pratique comique conventionnelle, interprète une des parties les plus visibles du corps masculin en termes de ce qu'il signifie pour la « sexualité » de l'individu qui se présente aux autres comme objet du regard.

Dans un sens, le Panurge du Tiers Livre est une figure de l'impuissance masculine, comme le montre clairement ici la formule de Frère Jean: «il n'y a pas grand chaleur dans les valées de [sa] braguette ». Face à cette accusation, qui fait souvent partie de l'amitié entre hommes, Panurge devient la victime d'une sorte de crise d'identité, puisque sa masculinité est mise en question. La réponse du personnage est on ne peut plus féroce, comme c'est le cas dans de nombreux passages du roman:

Et quand ma femme future seroit aussi gloutte du plaisir Venerien, que fut oncques Messalina, ou la marquise de Oinsestre en Angleterre, je te prie croire, que je l'ay encores plus copieux au contentement. Je ne ignore que Solomon dict, et en parloit comme clerc et sçavant: depuys luy Aristoteles a declairé l'estre des femmes estre de soy insatiable: mais je veulx qu'on saiche que de mesmes qualibre j'ay le ferrement infatiguable. Ne me allegue poinct icy en paragon les fabuleux ribaulx Hercules, Proculus Caesar, et Mahumet, qui se vente en son Alchoran avoir en ses genitoires la force de soixante guallefretiers. Il a menty le paillard. Ne me alleguez poinct l'Indian tant celebré par Theophraste, Pline, et Athenaeus, lequel avecques l'ayde de certaine herbe le faisoit en un jour soixante et dix fois et plus. Je n'en croy rien. Le nombre est supposé. Je te prie ne le croyre. Je te prie croyre (et ne croyras chose que ne soit vraye) mon naturel le sacre Ithyphalle messer Cotal d'Albingues, estre le prime d'el monde. (p. 436-437)

Panurge répète la configuration des attributs qui font partie du «compagnonnage » masculin jusqu'à nos jours : l'idée reçue de l'insatiabilité sexuelle de la femme, légitimée dans ce contexte par l'appel aux autorités canoniques, dont Salomon et Aristote; l'affirmation du caractère indubitable de la virilité de celui qui parle, accompagnée d'une mise en cause moqueuse de la façon dont tous les autres hommes exagèrent leur virilité à eux.

La masculinité chez Rabelais s'exhibe et se développe donc sur deux axes fondamentaux: d'abord, il y a la conception imaginaire du corps masculin qui est à la base de toutes ces manifestations discursives; deuxièmement, il y a l'intertexte très étendu manipulé par notre auteur, qui rend possible la médiatisation de l'imaginaire masculin à travers toutes sortes de références érudites. Autrement dit, les hommes dans ce contexte lisent toujours les corps de leurs contemporains mâles sous l'optique d'une herméneutique qui leur a été enseignée dans les écoles, et qui est largement responsable de la projection textuelle de leur sexe comme manière de lire, d'écrire et de raconter des histoires de corps, procédés qui sont au centre de leur conception de l'univers même. La masculinité chez Rabelais est donc la transcription d'un corps imaginaire déterminé dans son être par la notion 
fantastique de son sexe et de sa sexualité, qui déforme et structure à la fois les idées écrites qu'ont les hommes de toute l'histoire et de l'univers. Si le monde peut être contenu dans la bouche d'un de ces géants fictifs, il est clair aussi, du point de vue des hommes de l'époque de Rabelais, que le monde n'est littéralement qu'une manière de présenter, de voir, de lire, de raconter et d'écrire le corps masculin. L'écriture masculine rabelaisienne inscrit donc la demande adressée incessamment par des hommes à d'autres hommes de produire et de reproduire des discours conventionnels à propos de cette perception déformée et médiatisée du corps masculin et de son sexe - dans le sens anatomique et social de ce mot -, base d'une élaboration textuelle de l'être des hommes.

David LaGuardia Dartmouth College, Hanover, Etats-Unis 